\title{
A Table of Elliptic Integrals of the Second Kind*
}

\author{
By B. C. Carlson
}

\begin{abstract}
By evaluating elliptic integrals in terms of standard $R$-functions instead of Legendre's integrals, many (in one case 144) formulas in previous tables are unified. The present table includes only integrals of the first and second kinds having integrands with real singular points. The 216 integrals of this type listed in Gradshteyn and Ryzhik's table constitute a small fraction of the special cases of 13 integrals evaluated here. The interval of integration is not required, as it is in previous tables, to begin or end at a singular point of the integrand. Fortran codes for the standard $R$-functions are included in a Supplement.
\end{abstract}

\section{Introduction. Let}

$$
[p]=\left[p_{1}, p_{2}, \ldots, p_{n}\right]=\int_{y}^{x}\left(a_{1}+b_{1} t\right)^{p_{1} / 2} \cdots\left(a_{n}+b_{n} t\right)^{p_{n} / 2} d t,
$$

where $p_{1}, \ldots, p_{n}$ are nonzero integers, the integrand is real, and the integral is assumed to be well defined. Many integrals like

$$
\int\left(1-k^{2} \sin ^{2} \phi\right)^{p_{1} / 2} d \phi \text { and } \int\left(a+b z^{2}\right)^{p_{1} / 2}\left(c+d z^{2}\right)^{p_{2} / 2} d z
$$

can be put in the form (1.1) by letting $t=\sin ^{2} \phi$ or $t=z^{2}$.

For purposes of classification we assume the $b$ 's are nonzero and no two of the quantities $a_{i}+b_{i} t$ are proportional. If at most two $p$ 's are odd, the integral (1.1) is elementary. If exactly three $p$ 's are odd (the "cubic case"), the integral is elliptic of the first or second kind if all the even $p$ 's are positive, and otherwise it is third kind. The only such integral of the first kind is $[-1,-1,-1]$. If exactly four $p$ 's are odd (the "quartic case"), the integral is elliptic of the first or second kind if all the even $p$ 's are positive and $p_{1}+\cdots+p_{n} \leqslant-4$; otherwise it is third kind. The only such integral of the first kind is $[-1,-1,-1,-1]$. If more than four $p$ 's are odd, the integral is hyperelliptic.

Integrals of the first kind are traditionally expressed in terms of Legendre's $F(\phi, k)$ with $0 \leqslant k \leqslant 1$ and $0 \leqslant \phi \leqslant \pi / 2$. Integrals of the second kind require

Received October 6, 1986.

1980 Mathematics Subject Classification (1985 Revision). Primary 33A25; Secondary 33A30.

* Part of this work was done at the University of Maryland, where the author was a visitor at the Institute for Physical Science and Technology, with the support of AROD contract DAAG 29-80-C-0032. The rest was done in the Ames Laboratory, which is operated for the U. S. Department of Energy by Iowa State University under contract no. W-7405-ENG-82. The work was supported by the Director of Energy Research, Office of Basic Energy Sciences. 
$E(\phi, k)$ and usually $F$ also. We shall replace $F$ by the symmetric integral

$$
R_{F}(x, y, z)=\frac{1}{2} \int_{0}^{\infty}[(t+x)(t+y)(t+z)]^{-1 / 2} d t
$$

and $E$ by

$$
R_{D}(x, y, z)=\frac{3}{2} \int_{0}^{x}(t+x)^{-1 / 2}(t+y)^{-1 / 2}(t+z)^{-3 / 2} d t .
$$

These $R$-functions are homogeneous:

$$
\begin{aligned}
& R_{F}(\lambda x, \lambda y, \lambda z)=\lambda^{-1 / 2} R_{F}(x, y, z), \\
& R_{D}(\lambda x, \lambda y, \lambda z)=\lambda^{-3 / 2} R_{D}(x, y, z),
\end{aligned}
$$

and they are normalized so that

$$
R_{F}(x, x, x)=x^{-1 / 2}, \quad R_{D}(x, x, x)=x^{-3 / 2} .
$$

Fortran codes [6] for computing $R_{F}$ and $R_{D}$ when $x, y, z$ are real and nonnegative are listed in the Supplements section of this issue and can be found also in most of the major software libraries.

Customary integral tables [1], [7], [9] assume that the interval of integration begins or ends at a branch point of the integrand, and many special cases are listed according to the positions of the other branch points relative to the interval of integration and to one another. If the integral at hand does not have either limit of integration at a branch point, it must be split into two parts that do. In the present paper these two parts are recombined by the addition theorem, and the need to specify the relative positions of the branch points then disappears. The use of $R$-functions greatly facilitates the application of the addition theorem and leads to a further unification that cannot be achieved with Legendre's integrals, because the expressions for $R_{F}(x, y, z)$ and $R_{D}(x, y, z)$ in terms of Legendre's integrals with $0 \leqslant k \leqslant 1$ and $0 \leqslant \phi \leqslant \pi / 2$ depend on the relative sizes of $x, y$, and $z$ (see [5, (4.1), (4.2)], (5.25), and (5.32)).

Integrals of the third kind and integrands with conjugate complex branch points, resulting from an irreducible quadratic factor $a_{i}+b_{i} t+c_{i} t^{2}$, will be deferred to later papers. (Integrals of the first kind with quadratic factors are treated in [3].) The main table in Section 2 consists of quartic cases, since cubic cases can be obtained from these by putting $a_{i}=1$ and $b_{i}=0$ for various choices of $i$. To select integrals that are relatively simple and occur most commonly in practice, we arbitrarily require $\sum\left|p_{i}\right| \leqslant 8$. Apart from permutation of subscripts in (1.1), there are just nine quartic cases of the first or second kind satisfying this criterion: $[-1,-1,-1,-1]$, $[1,-1,-1,-3],[-1,-1,-1,-3,2],[-1,-1,-3,-3],[1,-1,-3,-3],[1,1,-3,-3]$, $[-1,-1,-1,-5],[1,-1,-1,-5]$, and $[1,1,-1,-5]$. The integral $[-1,-1,-1,-3]$ is a special case of $[-1,-1,-1,-3,2]$ with $a_{5}=1$ and $b_{5}=0$.

Section 3 presents four cubic cases not contained in the nine quartic cases: $[3,-1,-3],[3,-1,-1],[-3,-3,-3]$, and $[1,1,1]$.

The method of evaluating the integrals is discussed in Sections 4 and 5. The fundamental integrals are $[-1,-1,-1,-1]$ and $[1,-1,-1,-3]$, and the rest are obtained from these by recurrence relations. The single formula (2.7) for $[1,-1,-1,-3]$ replaces 72 cases occupying the nine pages of $\$ 3.168$ in Gradshteyn and Ryzhik’s 
table [7], as well as 72 cubic cases: 18 cases of $[-1,-1,-3]$ in $\$ 3.133,18$ cases of $[1,-1,-1]$ in $\S 3.141$, and 36 cases of $[1,-1,-3]$ in $\S 3.142$.

By using [5, (4.1), (4.2)], (2.6) was checked against formulas $1,3,5,7$ of [7, §3.147], and (2.7) was checked against formulas $1,5,42,70$ of $[7, \S 3.168]$. The nine integrals in Section 2 and the four in Section 3 were checked numerically to $6 \mathrm{~S}$ for $y=0.5$, $x=2.0, a_{i}=0.5+i, b_{i}=2.5-i$ by the SLATEC numerical quadrature routine QNG and the routines for $R_{F}$ and $R_{D}$ in the Supplements section of this issue.

2. Table of Quartic Cases. We assume $x>y$ and $a_{i}+b_{i} t>0, y<t<x$, for all $i$, and we define

$$
\begin{gathered}
d_{i j}=a_{i} b_{j}-a_{j} b_{i}, \\
X_{i}=\left(a_{i}+b_{i} x\right)^{1 / 2}, \quad Y_{i}=\left(a_{i}+b_{i} y\right)^{1 / 2}, \\
U_{i j}=\left(X_{i} X_{j} Y_{k} Y_{m}+Y_{i} Y_{j} X_{k} X_{m}\right) /(x-y),
\end{gathered}
$$

where $i, j, k, m$ is any permutation of $1,2,3,4$. These definitions imply

$$
U_{i k}^{2}-U_{i m}^{2}=d_{i j} d_{k m}
$$

and consequently the arguments of the $R$-functions appearing in the table differ by quantities independent of $x$ and $y$. If one limit of integration is infinite, (2.3) simplifies to

$$
\begin{aligned}
& U_{i j}=\left(b_{i} b_{j}\right)^{1 / 2} Y_{k} Y_{m}+Y_{i} Y_{j}\left(b_{k} b_{m}\right)^{1 / 2}, \\
& U_{i j}=X_{i} X_{j}\left(b_{k} b_{m}\right)^{1 / 2}+\left(b_{i} b_{j}\right)^{1 / 2} X_{k} X_{m}, \quad y=+\infty,
\end{aligned}
$$

all square roots being nonnegative.

If one limit of integration is a branch point of the integrand, then $X_{i}$ or $Y_{i}$ will be 0 for some value of $i$ (with $p_{i} \geqslant-1$ since we assume that the integral exists), and one of the two terms in every $U_{i j}$ will vanish. If both limits of integration are branch points, the elliptic integral is called complete, and one of the $U_{i j}$ will be 0 . It is not assumed that $b_{i} \neq 0$ nor that $d_{i j} \neq 0$ unless one of these quantities occurs in a denominator. The relation $d_{i j}=0$ is equivalent to proportionality of $a_{i}+b_{i} t$ and $a_{j}+b_{j} t$. The nine quartic cases listed in Section 1 follow. Only the first two are treated by Gradshteyn and Ryzhik [7, §3.147, §3.168].

$$
\begin{gathered}
\int_{y}^{x}\left[\left(a_{1}+b_{1} t\right)\left(a_{2}+b_{2} t\right)\left(a_{3}+b_{3} t\right)\left(a_{4}+b_{4} t\right)\right]^{-1 / 2} d t \\
=2 R_{F}\left(U_{12}^{2}, U_{13}^{2}, U_{14}^{2}\right) . \\
\int_{y}^{x}\left(a_{1}+b_{1} t\right)^{1 / 2}\left[\left(a_{2}+b_{2} t\right)\left(a_{3}+b_{3} t\right)\right]^{-1 / 2}\left(a_{4}+b_{4} t\right)^{-3 / 2} d t \\
=\frac{2}{3} d_{12} d_{13} R_{D}\left(U_{12}^{2}, U_{13}^{2}, U_{14}^{2}\right)+\frac{2 X_{1} Y_{1}}{X_{4} Y_{4} U_{14}} .
\end{gathered}
$$


The next equation remains valid even if $a_{5}+b_{5} t$ changes sign in the interval of integration.

$$
\begin{aligned}
& \int_{1}^{x}\left[\left(a_{1}+b_{1} t\right)\left(a_{2}+b_{2} t\right)\left(a_{3}+b_{3} t\right)\right]^{-1 / 2}\left(a_{4}+b_{4} t\right)^{-3 / 2}\left(a_{5}+b_{5} t\right) d t \\
& =\frac{2 d_{12} d_{13} d_{54}}{3 d_{14}} R_{D}\left(U_{12}^{2}, U_{13}^{2}, U_{14}^{2}\right) \\
& +\frac{2 d_{15}}{d_{14}} R_{F}\left(U_{12}^{2}, U_{13}^{2}, U_{14}^{2}\right)+\frac{2 d_{54} X_{1} Y_{1}}{d_{14} X_{4} Y_{4} U_{14}} \\
& \int_{y}^{x}\left[\left(a_{1}+b_{1} t\right)\left(a_{2}+b_{2} t\right)\right]^{-1 / 2}\left[\left(a_{3}+b_{3} t\right)\left(a_{4}+b_{4} t\right)\right]^{-3 / 2} d t \\
& =\frac{2}{3 d_{34}^{2}}\left(b_{3}^{2} d_{14} d_{24}+b_{4}^{2} d_{13} d_{23}\right) R_{D}\left(U_{13}^{2}, U_{14}^{2}, U_{12}^{2}\right) \\
& -\frac{4 b_{3} b_{4}}{d_{34}^{2}} R_{F}\left(U_{12}^{2}, U_{13}^{2}, U_{14}^{2}\right)+\frac{2}{d_{34}^{2} U_{12}}\left(\frac{b_{3}^{2} X_{4} Y_{4}}{X_{3} Y_{3}}+\frac{b_{4}^{2} X_{3} Y_{3}}{X_{4} Y_{4}}\right) \\
& \int_{y}^{x}\left(a_{1}+b_{1} t\right)^{1 / 2}\left(a_{2}+b_{2} t\right)^{-1 / 2}\left[\left(a_{3}+b_{3} t\right)\left(a_{4}+b_{4} t\right)\right]^{-3 / 2} d t \\
& =\frac{2 d_{13} d_{14}}{3 d_{34}^{2}}\left(b_{3} d_{24}+b_{4} d_{23}\right) R_{D}\left(U_{13}^{2}, U_{14}^{2}, U_{12}^{2}\right) \\
& -\frac{2}{d_{34}^{2}}\left(b_{3} d_{14}+b_{4} d_{13}\right) R_{F}\left(U_{12}^{2}, U_{13}^{2}, U_{14}^{2}\right) \\
& +\frac{2 b_{3} d_{13} X_{4} Y_{4}}{d_{34}^{2} U_{12} X_{3} Y_{3}}+\frac{2 b_{4} d_{14} X_{3} Y_{3}}{d_{34}^{2} U_{12} X_{4} Y_{4}} \\
& \int_{1}^{x}\left[\left(a_{1}+b_{1} t\right)\left(a_{2}+b_{2} t\right)\right]^{1 / 2}\left[\left(a_{3}+b_{3} t\right)\left(a_{4}+b_{4} t\right)\right]^{-3 / 2} d t \\
& =\frac{4 d_{12} d_{13} d_{24}}{3 d_{34}} R_{D}\left(U_{12}^{2}, U_{13}^{2}, U_{14}^{2}\right)+\frac{2 d_{12}}{d_{34}} R_{F}\left(U_{12}^{2}, U_{13}^{2}, U_{14}^{2}\right) \\
& +\frac{2}{d_{34} U_{14}}\left(\frac{d_{24} X_{1} Y_{1}}{X_{4} Y_{4}}-\frac{d_{13} X_{2} Y_{2}}{X_{3} Y_{3}}\right) \\
& \int_{y}^{x}\left[\left(a_{1}+b_{1} t\right)\left(a_{2}+b_{2} t\right)\left(a_{3}+b_{3} t\right)\right]^{-1 / 2}\left(a_{4}+b_{4} t\right)^{-5 / 2} d t \\
& =\frac{-4 b_{4}}{9 d_{14}}\left(\frac{b_{1}}{d_{14}}+\frac{b_{2}}{d_{24}}+\frac{b_{3}}{d_{34}}\right)\left\{d_{12} d_{13} R_{D}\left(U_{12}^{2}, U_{13}^{2}, U_{14}^{2}\right)+\frac{3 X_{1} Y_{1}}{X_{4} Y_{4} U_{14}}\right\} \\
& +\frac{2}{3}\left(\frac{2 b_{1}^{2}}{d_{14}^{2}}+\frac{b_{1} b_{2}}{d_{14} d_{24}}+\frac{b_{1} b_{3}}{d_{14} d_{34}}-\frac{b_{2} b_{3}}{d_{24} d_{34}}\right) R_{F}\left(U_{12}^{2}, U_{13}^{2}, U_{14}^{2}\right) \\
& -\frac{2 b_{4}^{2}}{3 d_{14} d_{24} d_{34}}\left(X_{1} X_{2} X_{3} X_{4}^{-3}-Y_{1} Y_{2} Y_{3} Y_{4}^{-3}\right) \text {. }
\end{aligned}
$$




$$
\begin{aligned}
\int_{y}^{x}\left(a_{1}+\right. & \left.b_{1} t\right)^{1 / 2}\left[\left(a_{2}+b_{2} t\right)\left(a_{3}+b_{3} t\right)\right]^{-1 / 2}\left(a_{4}+b_{4} t\right)^{-5 / 2} d t \\
= & \frac{2}{9}\left(\frac{b_{1}}{d_{14}}-\frac{2 b_{2}}{d_{24}}-\frac{2 b_{3}}{d_{34}}\right)\left\{d_{12} d_{13} R_{D}\left(U_{12}^{2}, U_{13}^{2}, U_{14}^{2}\right)+\frac{3 X_{1} Y_{1}}{X_{4} Y_{4} U_{14}}\right\} \\
& -\frac{2 b_{4} d_{12} d_{13}}{3 d_{14} d_{24} d_{34}} R_{F}\left(U_{12}^{2}, U_{13}^{2}, U_{14}^{2}\right) \\
& -\frac{2 b_{4}}{3 d_{24} d_{34}}\left(X_{1} X_{2} X_{3} X_{4}^{-3}-Y_{1} Y_{2} Y_{3} Y_{4}^{-3}\right) . \\
\int_{y}^{x}\left[\left(a_{1}+\right.\right. & \left.\left.b_{1} t\right)\left(a_{2}+b_{2} t\right)\right]^{1 / 2}\left(a_{3}+b_{3} t\right)^{-1 / 2}\left(a_{4}+b_{4} t\right)^{-5 / 2} d t \\
= & \frac{-2}{9 d_{14} d_{34}}\left(d_{13} d_{24}+d_{23} d_{14}\right)\left\{d_{12} d_{13} R_{D}\left(U_{12}^{2}, U_{13}^{2}, U_{14}^{2}\right)+\frac{3 X_{1} Y_{1}}{X_{4} Y_{4} U_{14}}\right\} \\
& -\frac{2 d_{12} d_{13}}{3 d_{14} d_{34}} R_{F}\left(U_{12}^{2}, U_{13}^{2}, U_{14}^{2}\right) \\
& -\frac{2}{3 d_{34}}\left(X_{1} X_{2} X_{3} X_{4}^{-3}-Y_{1} Y_{2} Y_{3} Y_{4}^{-3}\right) .
\end{aligned}
$$

3. Cubic Cases. By putting $a_{i}=1$ and $b_{i}=0$ for various choices of $i, 13$ cubic cases can be evaluated from the quartic cases in Section 2 and do not need to be listed separately. Eight of these are given by Gradshteyn and Ryzhik [7, $\S \S 3.131-3.135,3.141,3.142]:[-1,-1,-1],[-1,-1,-1,2],[-1,-1,-3],[-1,-1,-5]$, $[-1,-3,-3],[1,-1,-1],[1,1,-1]$, and $[1,-1,-3]$. They do not give the other five: $[1,1,-3],[1,1,-5],[1,-1,-5],[1,-3,-3]$, and $[-1,-1,-3,2]$.

In this section we list four cubic cases not contained in the quartic cases of Section $2:[3,-1,-3],[3,-1,-1],[-3,-3,-3]$, and $[1,1,1]$. Only $[-3,-3,-3]$ is given by Gradshteyn and Ryzhik [7. \$3.136], and only two cases of this are listed. each with an infinite limit of integration, because the integral diverges if it begins or ends at a finite branch point with $p_{i}=-3$. If the closed interval of integration lies in the open interval between two finite branch points with $p_{i}=-3$, there is no way to evaluate the integral by using previous tables.

In place of (2.3) we define

$$
U_{i}=\left(X_{i} Y_{j} Y_{h}+Y_{i} X_{j} X_{k}\right) /(x-y)
$$

where $i, j, k$ is any permutation of $1,2,3$. Since this implies

$$
U_{i}^{2}-U_{i}^{2}=b_{k} d_{i j}
$$

the arguments of the $R$-functions in the table differ by quantities independent of $x$ and $y$. If one limit of integration is infinite. (3.1) simplifies to

$$
U_{i}=\left(b_{j} b_{k}\right)^{1 / 2} Y_{i} \quad \text { if } x=+\infty, \quad U_{i}=\left(b_{j} b_{h}\right)^{1 / 2} X_{i} \quad \text { if } y=-\infty
$$


the square roots being nonnegative. The remarks in the paragraph preceding (2.6) apply, after replacement of $U_{i j}$ by $U_{i}$, also to the following integrals.

$$
\begin{aligned}
& \int_{y}^{x}\left(a_{1}+b_{1} t\right)^{3 / 2}\left(a_{2}+b_{2} t\right)^{-1 / 2}\left(a_{3}+b_{3} t\right)^{-3 / 2} d t \\
& =\frac{2 d_{13}}{b_{3} d_{23}}\left\{\frac{1}{3} d_{12}\left(b_{1} d_{23}+b_{2} d_{13}\right) R_{D}\left(U_{2}^{2}, U_{3}^{2}, U_{1}^{2}\right)\right. \\
& \left.-d_{12} R_{F}\left(U_{1}^{2}, U_{2}^{2}, U_{3}^{2}\right)+\frac{d_{13} X_{2} Y_{2}}{X_{3} Y_{3} U_{1}}\right\}+\frac{2 b_{1} X_{1} Y_{1}}{b_{3} U_{1}} \\
& \int_{y}^{x}\left(a_{1}+b_{1} t\right)^{3 / 2}\left[\left(a_{2}+b_{2} t\right)\left(a_{3}+b_{3} t\right)\right]^{-1 / 2} d t \\
& =\frac{4}{3 b_{2} b_{3}}\left(b_{2} d_{13}+b_{3} d_{12}\right)\left\{\frac{1}{3} d_{12} d_{13} R_{D}\left(U_{2}^{2}, U_{3}^{2}, U_{1}^{2}\right)+\frac{X_{1} Y_{1}}{U_{1}}\right\} \\
& -\frac{2 d_{12} d_{13}}{3 b_{2} b_{3}} R_{F}\left(U_{1}^{2}, U_{2}^{2}, U_{3}^{2}\right)+\frac{2 b_{1}}{3 b_{2} b_{3}}\left(X_{1} X_{2} X_{3}-Y_{1} Y_{2} Y_{3}\right) \text {. } \\
& \int_{y}^{x}\left[\left(a_{1}+b_{1} t\right)\left(a_{2}+b_{2} t\right)\left(a_{3}+b_{3} t\right)\right]^{-3 / 2} d t \\
& =\frac{4 b_{1} b_{2}}{3 d_{12}}\left(\frac{b_{1} b_{2}}{d_{12}}+\frac{b_{2} b_{3}}{d_{23}}+\frac{b_{3} b_{1}}{d_{31}}\right) R_{D}\left(U_{1}^{2}, U_{2}^{2}, U_{3}^{2}\right) \\
& +\frac{2 b_{1} b_{2}}{d_{12}^{2}}\left(\frac{b_{1}}{d_{13}}+\frac{b_{2}}{d_{23}}\right) R_{F}\left(U_{1}^{2}, U_{2}^{2}, U_{3}^{2}\right)+\frac{2 b_{3}^{2}}{d_{13} d_{23} X_{3} Y_{3} U_{3}} \\
& -\frac{2}{d_{12}^{2} U_{3}}\left(\frac{b_{1}^{3} X_{2} Y_{2}}{d_{13} X_{1} Y_{1}}+\frac{b_{2}^{3} X_{1} Y_{1}}{d_{23} X_{2} Y_{2}}\right) \\
& =\sum \frac{2 b_{3}^{2}}{d_{13} d_{23}}\left\{\frac{1}{3} b_{1} b_{2} R_{D}\left(U_{1}^{2}, U_{2}^{2}, U_{3}^{2}\right)+\frac{1}{X_{3} Y_{3} U_{3}}\right\},
\end{aligned}
$$

where $\sum$ denotes summation over cyclic permutations of the subscripts $1,2,3$. The same notation is used in the next formula.

$$
\begin{aligned}
& \int_{y}^{x}\left[\left(a_{1}\right.\right.\left.\left.+b_{1} t\right)\left(a_{2}+b_{2} t\right)\left(a_{3}+b_{3} t\right)\right]^{1 / 2} d t \\
&= \frac{-2\left(\sum b_{1}^{2} d_{23}^{2}\right)}{15 b_{1} b_{2} b_{3}^{2}}\left\{\frac{1}{3} d_{13} d_{23} R_{D}\left(U_{1}^{2}, U_{2}^{2}, U_{3}^{2}\right)+\frac{X_{3} Y_{3}}{U_{3}}\right\} \\
&-\frac{2 d_{13} d_{23}}{15 b_{1} b_{2} b_{3}^{2}}\left(b_{1} d_{23}+b_{2} d_{13}\right) R_{F}\left(U_{1}^{2}, U_{2}^{2}, U_{3}^{2}\right) \\
&+\frac{2}{15} X_{1} X_{2} X_{3}\left(\frac{X_{1}^{2}}{b_{1}}+\frac{X_{2}^{2}}{b_{2}}+\frac{X_{3}^{2}}{b_{3}}\right)-\frac{2}{15} Y_{1} Y_{2} Y_{3}\left(\frac{Y_{1}^{2}}{b_{1}}+\frac{Y_{2}^{2}}{b_{2}}+\frac{Y_{3}^{2}}{b_{3}}\right) \\
&= \frac{2}{15} \sum\left\{\frac{-d_{12}^{2}}{b_{1} b_{2}}\left[\frac{1}{3} d_{13} d_{23} R_{D}\left(U_{1}^{2}, U_{2}^{2}, U_{3}^{2}\right)+\frac{X_{3} Y_{3}}{U_{3}}\right]\right. \\
&\left.+b_{1}^{-1}\left(X_{1}^{3} X_{2} X_{3}-Y_{1}^{3} Y_{2} Y_{3}\right)\right\} .
\end{aligned}
$$


4. The Two Fundamental Integrals. In this section we shall prove (2.6) and (2.7) for $[-1,-1,-1,-1]$ and $[1,-1,-1,-3]$, from which the remaining integrals can be obtained by the recurrence relations of Section 5. In order that the first part of the proof shall apply for future purposes to $[1,-1,-1,-1,-2]$, which is an integral of the third kind, we do not restrict the number $n$ of factors in (1.1) to be 4 . It will be important that these three integrals have $p_{1}>-2$ and $\sum p_{i}=-4$.

In (1.1) we assume $x>y$ and $a_{i}+b_{i} t>0, y<t<x$, for all $i$. In the notation of Section 2 this implies $X_{i}^{2} \geqslant 0$ and $Y_{i}^{2} \geqslant 0$ for all $i$. Temporarily we assume further that $-a_{1} / b_{1}>x$ and that $a_{i}+b_{i} t>0, y \leqslant t \leqslant-a_{1} / b_{1}$, for $i>1$. This assumption, which will later be removed by analytic continuation, means that $-a_{1} / b_{1}$ is the first singularity encountered to the right of the interval of integration. The first part of the assumption implies $\left(a_{1}+b_{1} x\right) / b_{1}<0$, whence $X_{1}^{2}>0, b_{1}<0$, and $Y_{1}^{2}>0$, since $Y_{1}^{2}=X_{1}^{2}-b_{1}(x-y)$. The second part of the assumption implies $a_{i}+$ $b_{i}\left(-a_{1} / b_{1}\right)>0$, whence $d_{1 i}>0, i>1$.

We can now split (1.1) into two parts, both well defined if $p_{1}>-2$ :

$$
\begin{aligned}
{[p]=} & \int_{y}^{-a_{1} / b_{1}} \prod_{i=1}^{n}\left(a_{i}+b_{i} t\right)^{p_{i} / 2} d t \\
& -\int_{x}^{-a_{1} / h_{1}} \prod_{i=1}^{n}\left(a_{i}+b_{i} t\right)^{p_{i} / 2} d t=I_{y}-I_{x} .
\end{aligned}
$$

It suffices to consider $I_{y}$ because $I_{x}$ is the same with $y$ replaced by $x$. The interval of integration is mapped onto the positive real line by a change of integration variable:

$$
\begin{aligned}
u=\frac{t-y}{Y_{1}^{2}\left(a_{1}+b_{1} t\right)}, & t=\frac{y+a_{1} Y_{1}^{2} u}{1-b_{1} Y_{1}^{2} u}, \\
\frac{d t}{d u}=\frac{Y_{1}^{4}}{\left(1-b_{1} Y_{1}^{2} u\right)^{2}}, & a_{i}+b_{i} t=\frac{Y_{1}^{2} d_{1 i} u+Y_{i}^{2}}{1-b_{1} Y_{1}^{2} u},
\end{aligned}
$$

where $d_{11}=0$. If $\sum p_{i}=-4$ the powers of $1-b_{1} Y_{1}^{2} u$ cancel, and we find

$$
\begin{aligned}
I_{y} & =Y_{1}^{4+p_{1}} \int_{0}^{\infty} \prod_{i=2}^{n}\left(Y_{1}^{2} d_{1 i} u+Y_{i}^{2}\right)^{p_{i} / 2} d u \\
& =\prod_{j=2}^{n}\left(d_{1 j}\right)^{p_{j} / 2} \int_{0}^{\infty} \prod_{i=2}^{n}\left(u+Y_{i}^{2} / Y_{1}^{2} d_{1 i}\right)^{p_{i} / 2} d u .
\end{aligned}
$$

The integral $I_{x}$ is the same with $Y_{i}^{2} / Y_{1}^{2} d_{1 i}$ replaced by $X_{i}^{2} / X_{1}^{2} d_{1 i}$, and the difference,

$$
X_{i}^{2} / X_{1}^{2} d_{1 i}-Y_{i}^{2} / Y_{1}^{2} d_{1 i}=(x-y) / X_{1}^{2} Y_{1}^{2},
$$

is positive and independent of $i$. Using the notation

$$
\lambda=(x-y) / X_{1}^{2} Y_{1}^{2}, \quad z_{i}=Y_{i}^{2} / Y_{1}^{2} d_{1 i}, \quad z_{i}+\lambda=X_{i}^{2} / X_{1}^{2} d_{1 i},
$$

we find from (4.1), (4.3), (1.2), and (1.3) that

$$
\begin{aligned}
& {[-1,-1,-1,-1]=} 2\left(d_{12} d_{13} d_{14}\right)^{-1 / 2} \\
& \cdot\left[R_{F}\left(z_{2}, z_{3}, z_{4}\right)-R_{F}\left(z_{2}+\lambda, z_{3}+\lambda, z_{4}+\lambda\right)\right] \\
& {[1,-1,-1,-3]=\frac{2}{3}\left(d_{12} d_{13}\right)^{-1 / 2}\left(d_{14}\right)^{-3 / 2} } \\
& \cdot\left[R_{D}\left(z_{2}, z_{3}, z_{4}\right)-R_{D}\left(z_{2}+\lambda, z_{3}+\lambda, z_{4}+\lambda\right)\right]
\end{aligned}
$$


The addition theorem [4. (9). (13)] for $R_{F}$ is

$$
\begin{gathered}
R_{F}\left(z_{2}, z_{3} . z_{4}\right)= \\
R_{F}\left(z_{2}+\lambda . z_{3}+\lambda . z_{4}+\lambda\right) \\
+R_{F}\left(z_{2}+\mu . z_{3}+\mu . z_{4}+\mu\right) . \\
\left.z_{i}+\mu=\lambda^{-2 !}\left[\left(z_{i}+\lambda\right) z_{j} z_{k}\right]^{1 / 2}+\left[z_{i}\left(z_{j}+\lambda\right)\left(z_{k}+\lambda\right)\right]^{1 / 2}\right\}^{2} .
\end{gathered}
$$

where $i, j . k$ is any permutation of $2,3,4$. Thus (4.5) becomes

$$
\begin{gathered}
\text { [-1.-1.-1.-1] }=2\left(d_{12} d_{13} d_{14}\right)^{-1 / 2} R_{F}\left(z_{2}+\mu . z_{3}+\mu . z_{4}+\mu\right) . \\
z_{1}+\mu=\frac{\left(X_{1} X_{i} Y_{j} Y_{k}+Y_{1} Y_{i} X_{j} X_{k}\right)^{2}}{d_{1 i} d_{1 j} d_{1 k}(x-y)^{2}}=\frac{U_{1 i}^{2}}{d_{12} d_{13} d_{14}} .
\end{gathered}
$$

By the homogeneity property (1.4) we find

$$
[-1,-1,-1,-1]=2 R_{F}\left(U_{12}^{2}, U_{13}^{2}, U_{14}^{2}\right) .
$$

which is the same as (2.6).

This removal of the $d$ 's from the arguments of $R_{F}$ is the critical step. As shown by (1.2). an argument of $R_{F}$ must not be negative, and so the functions on the right-hand side of (4.5) require the branch points to be ordered so that $d_{12} . d_{13}$. and $d_{14}$ are positive. To show that (4.10) holds without the assumption that $-a_{1} / b_{1}$ is the first singularity to the right of the interval of integration, we use analytic continuation in $b_{1}$ or more conveniently in $k$, where

$$
\begin{gathered}
w=X_{1}^{2}=a_{1}+b_{1} x . \quad b_{1}=\frac{w-Y_{1}^{2}}{x-y} . \\
a_{1}=\frac{x Y_{1}^{2}-y w}{x-y}, \quad \frac{-a_{1}}{b_{1}}=x+\frac{w(x-y)}{Y_{1}^{2}-w} .
\end{gathered}
$$

We fix $x . y Y_{i}>0,1 \leqslant i \leqslant n$, and $X_{i}>0,2 \leqslant i \leqslant n$. Then $a_{1}$ and $b_{1}$ are functions of $u$. and we can make $-a_{1} / b_{1}$ be the first singularity to the right of the interval of integration by choosing $x$ ' positive and sufficiently small. For such values of $n$ we have proved that $(4.10)$ is true. We shall show that both sides of $(4.10)$ are analytic in $w$ on the complex plane cut along the nonpositive real axis. It follows by the permanence of functional relations that (4.10) holds in the cut plane and in particular for all positive values of $n$. Therefore it holds for any real value of $-a_{1} / b_{1}$ outside the closed interval of integration. The last statement is immediately evident from the graph of $a_{1}+b_{1} t$ as a function of $t$. since $a_{1}+b_{1} y$ has been fixed and $w=a_{1}+b_{1} x$.

To prove analyticity, we recall that an $R$-function is analytic when each of its arguments lies in the plane cut along the nonpositive real axis [2, (6.8-6). Theorem $(6.8-1)$ ]. Since (2.3) shows that $U_{i j}=\alpha_{i j} n^{1 / 2}+\beta_{i j}$. where $\alpha_{i j}$ and $\beta_{i j}$ are positive. $U_{i}^{2}$ lies in the cut plane when $u$ does, and so the right-hand side of (4.10) is analytic in the cut $n$-plane. The left side is defined by (1.1), which can be rewritten, when $\sum p_{i}=-4$. as

$$
[p]=(x-y)\left(\prod_{i=1}^{n} Y_{i}^{p_{i}}\right) R_{-1}\left(\frac{-p_{1}}{2} \ldots \frac{-p_{n}}{2}: \frac{X_{1}^{2}}{Y_{1}^{2}} \ldots \frac{X_{n}^{2}}{Y_{n}^{2}}\right)
$$


by taking $s=(x-t) /(t-y)$ as a new variable of integration and using [2, $(6.8-6)]$. Since $Y_{1}^{2}$ is positive and $X_{1}^{2}=w$, the right side of (4.12) and the left side of (4.10) are analytic in the cut $w$-plane, and the proof of $(2.6)$ is complete.

A different proof of (2.6) was given in [3], but the present proof is adaptable to (2.7) with only minor changes. The addition theorem for $R_{D}$, obtained by putting $\rho=z$ in $[11,(8.11)]$, is

$$
\begin{aligned}
R_{D}\left(z_{2}, z_{3}, z_{4}\right)= & R_{D}\left(z_{2}+\lambda, z_{3}+\lambda, z_{4}+\lambda\right) \\
& +R_{D}\left(z_{2}+\mu, z_{3}+\mu, z_{4}+\mu\right) \\
& +3\left[z_{4}\left(z_{4}+\lambda\right)\left(z_{4}+\mu\right)\right]^{-1 / 2},
\end{aligned}
$$

where $\mu$ is the same as in (4.7). Thus (4.6) becomes

$$
\begin{aligned}
{[1,-1,-1,-3]=} & \frac{2}{3}\left(d_{12} d_{13}\right)^{-1 / 2}\left(d_{14}\right)^{-3 / 2} \\
& \cdot\left\{R_{D}\left(z_{2}+\mu, z_{3}+\mu, z_{4}+\mu\right)\right. \\
& \left.+3\left[z_{4}\left(z_{4}+\lambda\right)\left(z_{4}+\mu\right)\right]^{-1 / 2}\right\} .
\end{aligned}
$$

Substituting (4.4) and (4.9) and using the homogeneity property (1.4), we find (2.7). The temporary assumption about $-a_{1} / b_{1}$ can again be removed by the permanence of functional relations. In the first term on the right-hand side of (2.7), $d_{12}$ and $d_{13}$ are linear functions of $w=X_{1}^{2}$ by (2.1) and (4.11), and $R_{D}$ is analytic in the cut $w$-plane by the same reasoning that applied earlier to $R_{F}$. The second term also is analytic because $X_{1} / U_{14}=w^{1 / 2} /\left(\alpha_{14} w^{1 / 2}+\beta_{14}\right)$, where $\alpha_{14}$ and $\beta_{14}$ are positive. Since the left side of (2.7) is a special case of (4.12), the proof is complete.

5. Recurrence Relations. Let $e_{i}$ denote an $n$-tuple with 1 in the $i$ th place and 0 's elsewhere (for example, $\left[p+2 e_{1}\right]=\left[p_{1}+2, p_{2}, \ldots, p_{n}\right]$ ). We shall first list some relations between different integrals, then give their proofs, and finally show how they can be used to obtain all the integrals in the table from the two fundamental integrals (2.6) and (2.7). The most useful relation is

$$
d_{i j}[p]=b_{j}\left[p+2 e_{i}\right]-b_{i}\left[p+2 e_{j}\right]
$$

Two others, involving the quantity

$$
A(p)=\prod_{i=1}^{n} X_{i}^{p_{i}}-\prod_{i=1}^{n} Y_{i}^{p_{i}}
$$

are

$$
\sum_{i=1}^{n} p_{i} b_{i}\left[p-2 e_{i}\right]=2 A(p)
$$

and

$$
\left(p_{1}+\cdots+p_{n}+2\right) b_{i}[p]=\sum_{j=1}^{n} p_{j} d_{j i}\left[p-2 e_{j}\right]+2 A\left(p+2 e_{i}\right)
$$

The latter, which can be used to raise the value of $\sum p_{i}$, contains $n$ integrals since $d_{i i}=0$. 
Recurrence relations for a single $p_{i}$ depend on the value of $n$. For $n=3$ and $i, j, k$ any permutation of $1,2,3$, we have

$$
\begin{aligned}
\left(p_{1}+p_{2}+p_{3}\right. & +4) b_{j} b_{k}\left[p+2 e_{i}\right] \\
& +\left\{\left(p_{i}+p_{j}+2\right) b_{j} d_{k i}+\left(p_{i}+p_{k}+2\right) b_{k} d_{j i}\right\}[p] \\
& +p_{i} d_{j i} d_{k i}\left[p-2 e_{i}\right]=2 b_{i} A\left(p+2 e_{j}+2 e_{k}\right) .
\end{aligned}
$$

The analogous relation for $n=4$ and $i, j, k, m$ a permutation of $1,2,3,4$ is

$$
\begin{aligned}
\left(p_{1}+p_{2}+p_{3}\right. & \left.+p_{4}+6\right) b_{j} b_{k} b_{m}\left[p+4 e_{i}\right] \\
& +\sum\left(p_{i}+p_{j}+p_{k}+4\right) b_{j} b_{k} d_{m i}\left[p+2 e_{i}\right] \\
& +\sum\left(p_{i}+p_{j}+2\right) b_{j} d_{k i} d_{m i}[p]+p_{i} d_{j i} d_{k i} d_{m i}\left[p-2 e_{i}\right] \\
= & 2 b_{i}^{2} A\left(p+2 e_{j}+2 e_{k}+2 e_{m}\right),
\end{aligned}
$$

where $\sum$ denotes summation over cyclic permutations of $j, k, m$. This relation is especially useful if $\sum p_{i}=-6$, because the first term vanishes.

Equation (5.1) follows at once from the definition of $[p]$ and the identity

$$
d_{i j}=b_{j}\left(a_{i}+b_{i} t\right)-b_{i}\left(a_{j}+b_{j} t\right) \text {. }
$$

To prove (5.3) we integrate both sides of

$$
2 \frac{d}{d t} \prod_{i=1}^{n}\left(a_{i}+b_{i} t\right)^{p_{i} / 2}=\sum_{i=1}^{n} p_{i} b_{i}\left(a_{i}+b_{i} t\right)^{-1} \prod_{j=1}^{n}\left(a_{j}+b_{j} t\right)^{p_{j} / 2}
$$

with respect to $t$ over the interval $[y, x]$.

If $p$ is replaced by $p+2 e_{i},(5.3)$ becomes

$$
\left(p_{i}+2\right) b_{i}[p]+\sum_{\substack{j=1 \\ j \neq i}}^{n} p_{j} b_{j}\left[p+2 e_{i}-2 e_{j}\right]=2 A\left(p+2 e_{i}\right),
$$

and if $p$ is replaced by $p-2 e_{j},(5.1)$ becomes

$$
b_{j}\left[p+2 e_{i}-2 e_{j}\right]=b_{i}[p]-d_{j i}\left[p-2 e_{j}\right] \text {. }
$$

Substitution of (5.10) in (5.9) yields (5.4). To prove (5.5) we use (5.7) twice to write $b_{i}^{2}\left(a_{j}+b_{j} t\right)\left(a_{k}+b_{k} t\right)$ as a quadratic polynomial in $a_{i}+b_{i} t$, multiply by $\Pi\left(a_{r}+b_{r} t\right)^{p_{r} / 2}$, and integrate to get

$$
\begin{aligned}
b_{i}^{2}\left[p+2 e_{j}+2 e_{k}\right]= & b_{j} b_{k}\left[p+4 e_{i}\right]+\left(b_{j} d_{k i}+b_{k} d_{j i}\right)\left[p+2 e_{i}\right] \\
& +d_{j i} d_{k i}[p] .
\end{aligned}
$$

Next we replace $p$ by $p+2 e_{j}+2 e_{k}$ in (5.3) with $n=3$ and find

$$
\begin{aligned}
p_{i} b_{i}\left[p-2 e_{i}+2 e_{j}+2 e_{k}\right]+\left(p_{j}+2\right) b_{j}\left[p+2 e_{k}\right] \\
+\left(p_{k}+2\right) b_{k}\left[p+2 e_{j}\right]=2 A\left(p+2 e_{j}+2 e_{k}\right) .
\end{aligned}
$$

In the first term we substitute (5.11) with $p$ replaced by $p-2 e_{i}$; in the second and third terms we use (5.1) with or without replacement of $j$ by $k$. The result is (5.5), and (5.6) has a similar proof starting from $b_{i}^{3}\left(a_{j}+b_{j} t\right)\left(a_{k}+b_{k} t\right)\left(a_{m}+b_{m} t\right)$ as a cubic polynomial in $a_{i}+b_{i} t$.

The following special cases of (5.1) show how to obtain (2.8), (2.9), (2.10), and (2.11) from (2.6) and (2.7):

$$
d_{14}[-1,-1,-1,-3]=b_{4}[1,-1,-1,-3]-b_{1}[-1,-1,-1,-1],
$$




$$
\begin{gathered}
b_{4}[-1,-1,-1,-3,2]=d_{54}[-1,-1,-1,-3]+b_{5}[-1,-1,-1,-1], \\
d_{34}[-1,-1,-3,-3]=b_{4}[-1,-1,-1,-3]-b_{3}[-1,-1,-3,-1], \\
b_{3}[1,-1,-3,-3]=d_{13}[-1,-1,-3,-3]+b_{1}[-1,-1,-1,-3], \\
b_{3}[1,1,-3,-3]=d_{23}[1,-1,-3,-3]+b_{2}[1,-1,-1,-3] .
\end{gathered}
$$

We have omitted $p_{5}=0$ in the two integrals on the right-hand side of (5.14). In (5.15), $[-1,-1,-3,-1]$ is found by interchanging the subscripts 3 and 4 in formula (2.8) specialized to $[-1,-1,-1,-3]$. Letting $[p]=[-1,-1,-1,-3]$ and $i=4$ in (5.6), we get $[-1,-1,-1,-5]$ from $[-1,-1,-1,-3]$ and $[-1,-1,-1,-1]$, since the first term of (5.6) is 0 . Equations (2.12) and (2.13) then follow from two more special cases of (5.1):

$$
\begin{gathered}
b_{4}[1,-1,-1,-5]=d_{14}[-1,-1,-1,-5]+b_{1}[-1,-1,-1,-3], \\
b_{4}[1,1,-1,-5]=d_{24}[1,-1,-1,-5]+b_{2}[1,-1,-1,-3] .
\end{gathered}
$$

The formulas resulting from this procedure can sometimes be simplified with the help of various identities:

$$
\begin{gathered}
b_{i} X_{j}^{2}-b_{j} X_{i}^{2}=b_{i} Y_{j}^{2}-b_{j} Y_{i}^{2}=d_{j i}, \\
X_{i}^{2} Y_{j}^{2}-Y_{i}^{2} X_{j}^{2}=(x-y) d_{j i}, \\
\sum a_{i} d_{j k}=\sum b_{i} d_{j k}=\sum d_{i m} d_{j k}=0, \\
\sum X_{i}^{2} d_{j k}=\sum Y_{i}^{2} d_{j k}=0, \\
\sum d_{i j} U_{i j} X_{k} Y_{k}=0,
\end{gathered}
$$

where $\sum$ denotes summation over cyclic permutations of $i, j, k$. These identities are obtained from definitions (2.1) to (2.3). Equation (5.22) is used to prove (5.23) and (5.23) to prove (5.24). Since $R_{D}$, unlike $R_{F}$, is symmetric in only its first two arguments, another useful relation is

$$
\begin{aligned}
d_{1 j} d_{k i} R_{D}\left(U_{1 i}^{2}, U_{1 j}^{2}, U_{1 k}^{2}\right)= & d_{1 k} d_{i j} R_{D}\left(U_{1 i}^{2}, U_{1 k}^{2}, U_{1 j}^{2}\right) \\
& +3 R_{F}\left(U_{12}^{2}, U_{13}^{2}, U_{14}^{2}\right)-\frac{3 U_{1 i}}{U_{1 j} U_{1 k}},
\end{aligned}
$$

where $i, j, k$ is any permutation of $2,3,4$. This can be proved by using [5, (4.14)] to express both sides in terms of the symmetric functions $R_{G}$ and $R_{F}$ and simplifying with the help of (2.4).

The four cubic cases in Section 3 can be obtained from (5.1) and (5.4) as follows:

$$
\begin{gathered}
b_{3}[3,-1,-3]=d_{13}[1,-1,-3]+b_{1}[1,-1,-1], \\
b_{2}[3,-1,-1]=d_{12}[1,-1,-1]+b_{1}[1,1,-1], \\
d_{12}[-3,-3,-3]=b_{2}[-1,-3,-3]-b_{1}[-3,-1,-3], \\
5 b_{1}[1,1,1]=d_{21}[1,-1,1]+d_{31}[1,1,-1]+2 A(3,1,1) .
\end{gathered}
$$

Aside from permutation of indices, each integral on the right-hand side of these equations is among the 13 cubic cases listed in the first paragraph of Section 3. Equation (5.24) is replaced by two identities,

$$
\begin{gathered}
\sum d_{i j} U_{k} X_{k} Y_{k}=0, \\
b_{i} U_{i} X_{j} Y_{j}-b_{j} U_{j} X_{i} Y_{i}=d_{j i} U_{k},
\end{gathered}
$$


and (5.25) is replaced by

$$
\begin{aligned}
b_{j} d_{k i} R_{D}\left(U_{i}^{2}, U_{j}^{2}, U_{k}^{2}\right)= & b_{k} d_{i j} R_{D}\left(U_{i}^{2}, U_{k}^{2}, U_{j}^{2}\right) \\
& +3 R_{F}\left(U_{1}^{2}, U_{2}^{2}, U_{3}^{2}\right)-\frac{3 U_{i}}{U_{j} U_{k}} .
\end{aligned}
$$

In these three equations $i, j, k$ is any permutation of $1,2,3$, and $\sum$ denotes summation over cyclic permutations of $i, j, k$. Equation (5.23) is used to prove (5.30), and (5.20) to prove (5.31). Equation (5.32) is proved in the same way as (5.25) except that (3.2) is used in place of (2.4).

Department of Mathematics

Iowa State University

Ames, Iowa 50011

1. P. F. Byrd \& M. D. Friedman, Handbook of Elliptic Integrals for Engineers and Scientists, 2nd ed., Springer-Verlag, New York, 1971.

2. B. C. CARlson, Special Functions of Applied Mathematics, Academic Press, New York, 1977.

3. B. C. Carlson, "Elliptic integrals of the first kind," SIA M J. Math. Anal., v. 8, 1977, pp. 231-242.

4. B. C. Carlson, "Short proofs of three theorems on elliptic integrals," SIAM J. Math. Anal., v. 9, 1978 , pp. 524-528.

5. B. C. Carlson, “Computing elliptic integrals by duplication,” Numer. Math., v. 33, 1979, pp. 1-16.

6. B. C. Carlson \& Elaine M. Notis, "AlgORITHM 577, Algorithms for incomplete elliptic integrals," ACM Trans. Math. Software, v. 7, 1981, pp. 398-403.

7. I. S. Gradshteyn \& I. M. RyzhiK, Table of Integrals, Series, and Products, Academic Press, New York, 1980.

8. D. H. LehMer, "The lemniscate constant," Math. Comp., v. 3, 1948/49, pp. 550-551.

9. A. P. Prudnikov, YU. A. BrychKov \& O. I. Marichev, Integrals and Series, vol. 1, Gordon and Breach, New York, 1986.

10. JoHN TodD, "The lemniscate constants," Comm. ACM, v. 18, 1975, pp. 14-19, 462.

11. D. G. Zill \& B. C. Carlson, "Symmetric elliptic integrals of the third kind," Math. Comp., v. 24, 1970, pp. 199-214. 


\title{
Supplement to A Table of Elliptic Integrals of the Second Kind
}

\author{
By B. C. Carlson
}

This supplement contains Fortran routines for the standard functions $R_{F}(x, y, z)$ and $R_{D}(x, y, z)$, followed by two examples of their use in computing elliptic integrals.

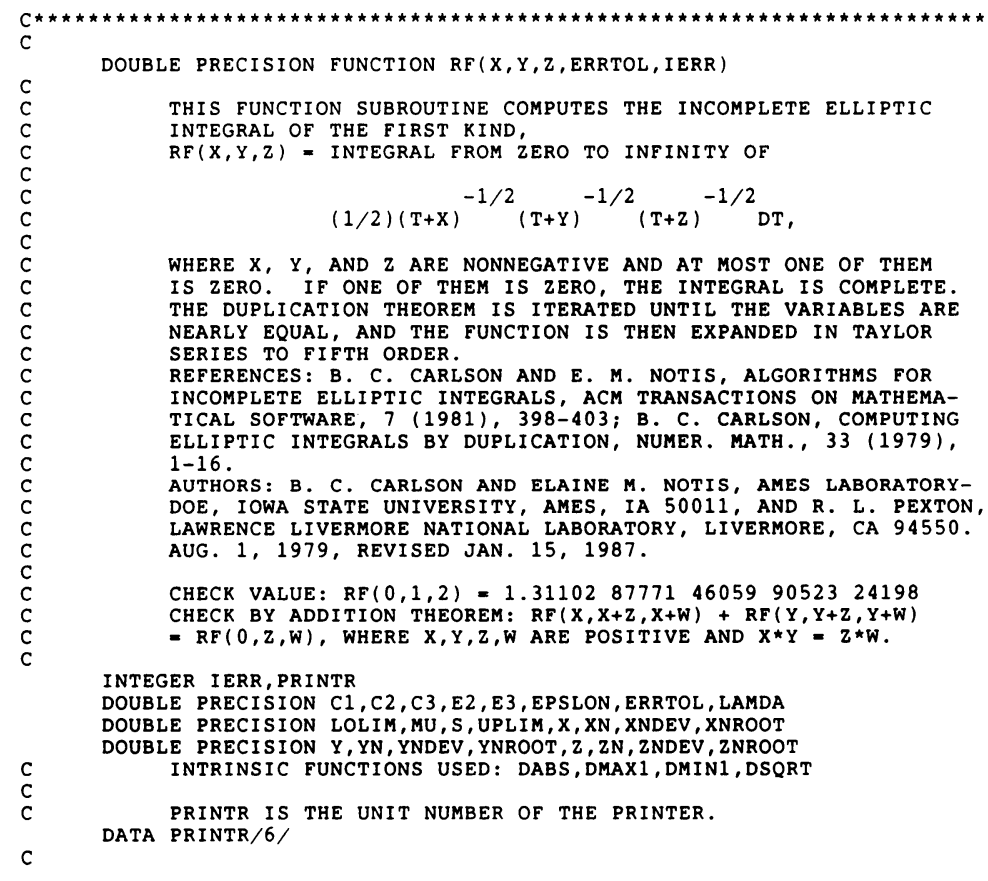




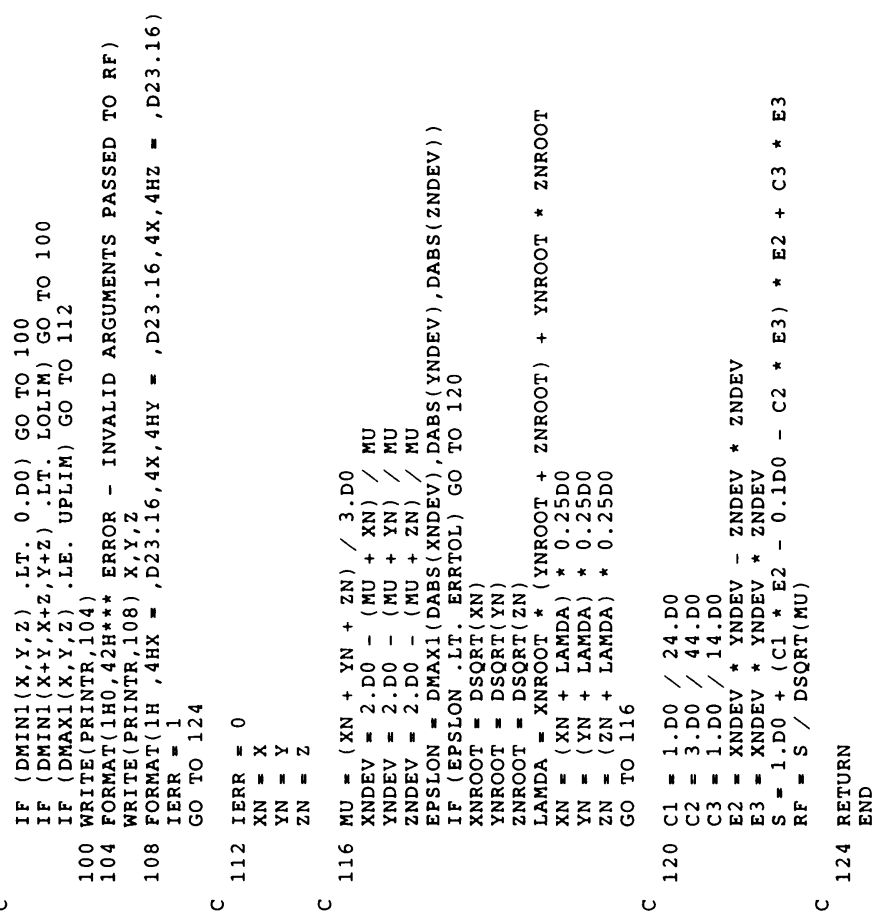

0uvo
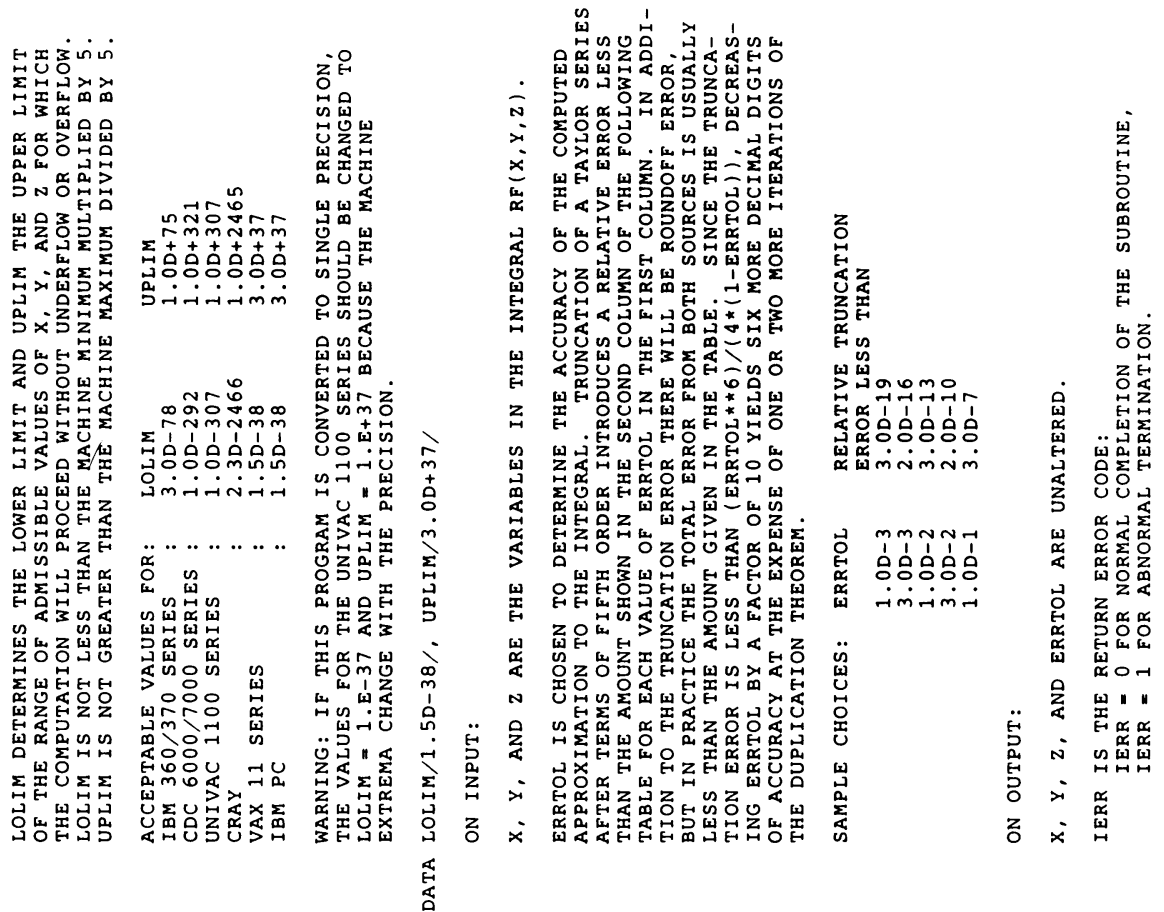


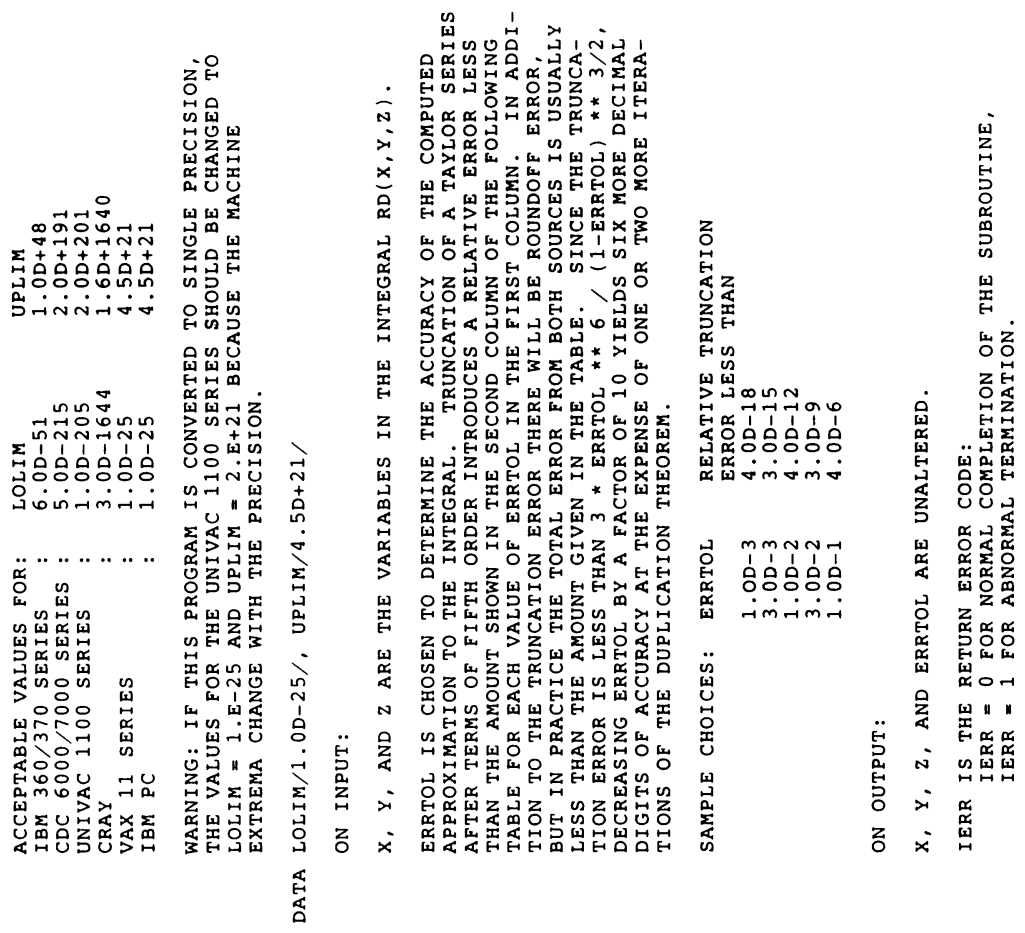

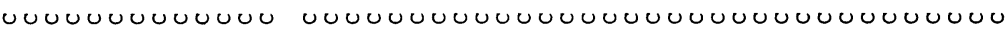

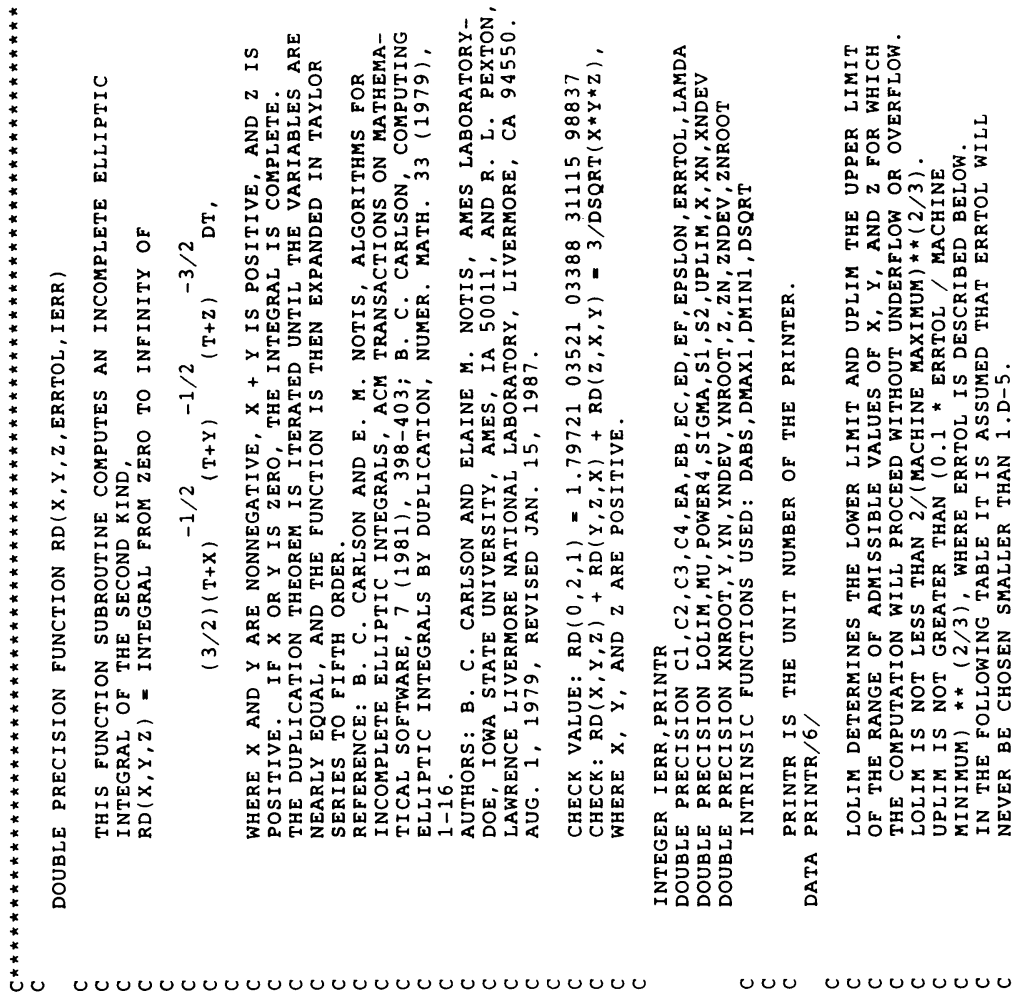



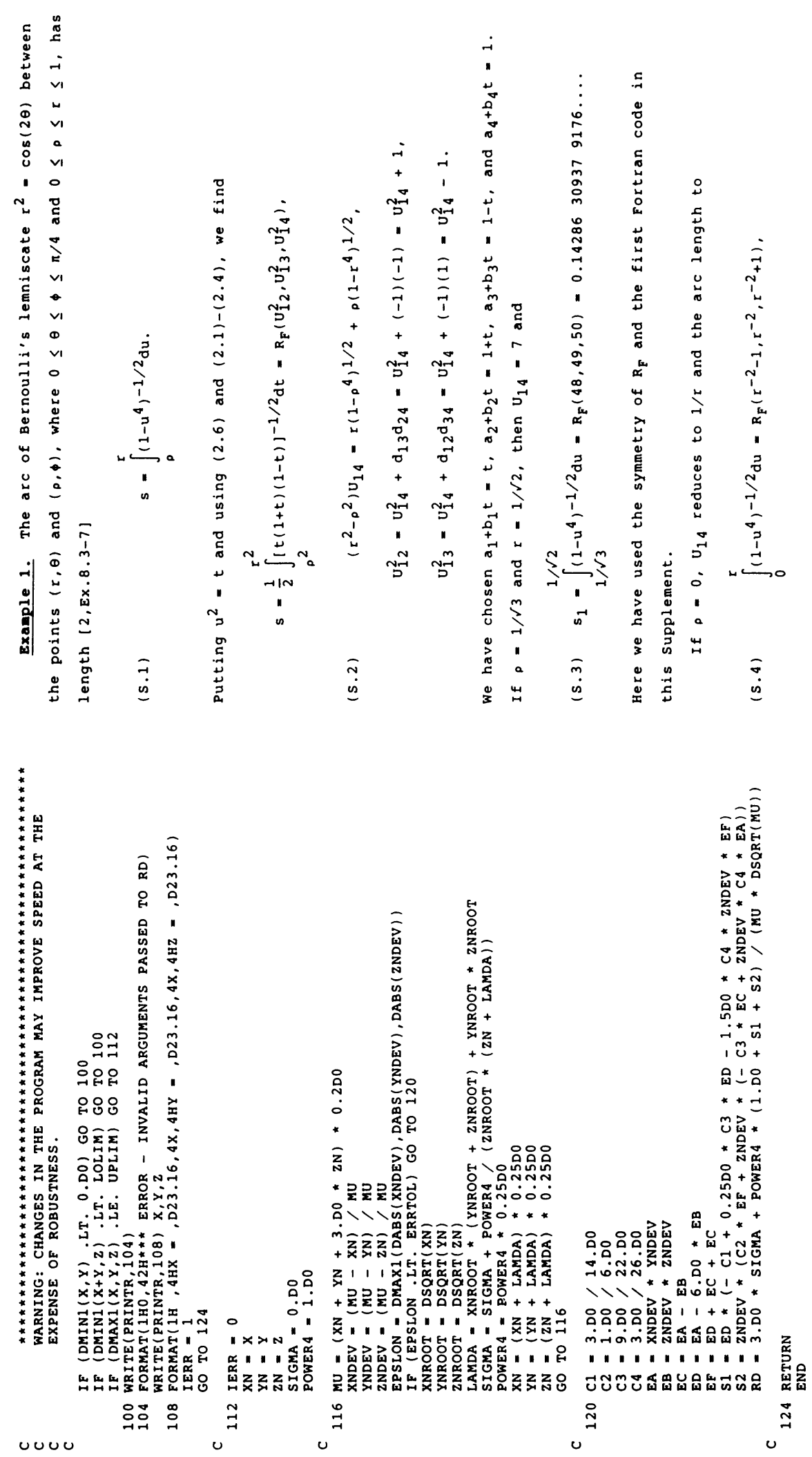

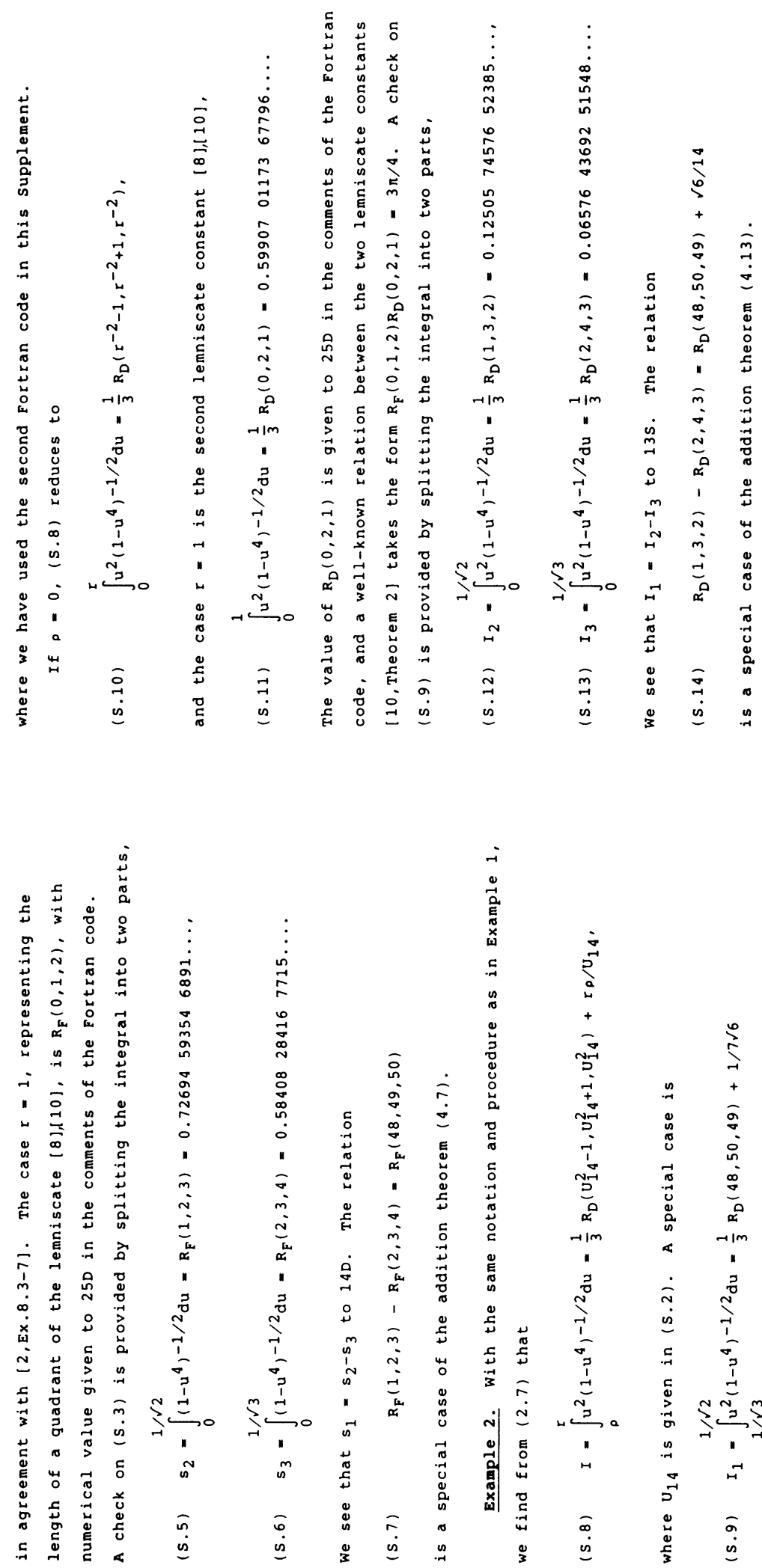